\title{
Study on the Chalkiness Character of Giant Embryo Rice and Observation on Its Microstructure in Grains
}

\author{
Bo Peng ${ }^{1}, \mathrm{Kun} \mathrm{He}^{1}$, Kun Xu${ }^{1}$, Dong-Ying Tang ${ }^{1}$, Juan Peng ${ }^{2}$, Xiao-Hua Song ${ }^{3}$, Xia-Yu Tian ${ }^{1}$, Yan-Fang Sun ${ }^{1}$, \\ Rui-Hua Pang ${ }^{1}$, Meng-Yang Zheng ${ }^{1}$, Hui-Long Li ${ }^{3}$, Gui-Ying Guo ${ }^{3}$, Xin-Hua Huang ${ }^{1}$, Quan-Xiu Wang ${ }^{1}$, \\ Wei Zhou ${ }^{1} \&$ Hong-Yu Yuan ${ }^{1}$ \\ ${ }^{1}$ College of Life Sciences and Institute for Conservation and Utilization of Agro-bioresources in Dabie \\ Mountains, Xinyang Normal University, Xinyang, China \\ ${ }^{2}$ Xinyang Station of Plant Protection and Inspection, Xinyang, China \\ ${ }^{3}$ Xinyang Academy of Agricultural Science, Xinyang, China \\ Correspondence: Bo Peng, College of Life Sciences and Institute for Conservation and Utilization of \\ Agro-bioresources in Dabie Mountains, Xinyang Normal University, Xinyang 464000, China. E-mail: \\ pengbo@xynu.edu.cn \\ Quan-Xiu Wang, College of Life Sciences and Institute for Conservation and Utilization of Agro-bioresources in \\ Dabie Mountains, Xinyang Normal University, Xinyang 464000, China. E-mail: wwqqxx08@163.com \\ Hong-Yu Yuan, College of Life Sciences and Institute for Conservation and Utilization of Agro-bioresources in \\ Dabie Mountains, Xinyang Normal University, Xinyang 464000, China. E-mail: yhongyu92@163.com
}

Received: April 19, 2020

doi:10.5539/jas.v12n8p171
Accepted: June 23, 2020

Online Published: July 15, 2020

URL: https://doi.org/10.5539/jas.v12n8p171

\begin{abstract}
Grain chalkiness greatly affects the grain appearance and milling, eating, cooking, and nutritional qualities, thus it is one of the most important traits of grain qualities. Based on the study of chalkiness characters in endosperm of different giant embryo rice, the relationship between the differences of chalkiness characters in endosperm of different giant embryo rice and the morphological structure, arrangement and development of starch granules in endosperm was clarified, which will lay a solid foundation for further revealing the formation mechanism of chalkiness characters in giant embryo rice and cultivating excellent new varieties of giant embryo rice. In this study, the chalkiness of endosperm (including chalkiness rate, chalkiness degree and chalkiness area) of 16 different rice varieties were investigated and analyzed. The results showed that the chalkiness of endosperm was closely related to the morphological structure and arrangement of starch granules in endosperm cells of different kinds of giant embryo rice, and there were significant differences between the chalkiness of different parts of the same giant embryo rice and the morphological structure and arrangement of starch granules in endosperm cells. Therefore, the results of this study will provide important information for the improvement of quality characters and the breeding of new rice varieties.
\end{abstract}

Keywords: giant embryo rice, chalkiness, endosperm, starch granule, scanning electron microscopy

\section{Introduction}

Rice (Oryza sativa L.) is one of the most important crops in the world. More than half of the world's population uses rice as its main food. As the largest country of rice production and consumption, rice planting and processing has made an important contribution to the healthy and sustainable development of China's economy. With the increase of the world population and the gradual improvement of people's living standards, the market demand for rice, especially for high-quality rice, is also increasing (Tian et al., 2009; Kim et al., 2013; Zheng et al., 2012). Therefore, the grian quality of rice determines the competitive position and price of rice in the consumer market.

Rice quality is a complex quantitative character, which is generally considered to include four aspects, namely cooking and eating quality, appearance quality, grinding and processing quality and nutritional quality (Peng et al., 2016). Appearance quality of rice includes grain length, grain width, grain thickness, length width ratio and chalkiness or transparency; Milling quality of rice mainly includes brown rice rate, milled rice rate and whole milled rice rate; Cooking and eating quality mainly includes amylose content, gel consistency and gelatinization 
temperature; Nutrition quality mainly includes protein content, amino acid content, fatty acid content and various trace elements, etc. (Hu et al., 2003; Zhang et al., 2013). The results of previous studies show that the appearance of chalkiness will reduce the appearance quality of rice and have a great negative impact on farmers' preference for rice varieties (Feng et al., 2017). Moreover, the grains with chalkiness are easy to be broken in the process of rice processing, which will greatly reduce the rate of whole rice, reduce the yield of rice, and affect the commodity value of rice.

The formation of chalkiness always affects grain quality traits in rice, especially the appearance quality, which is the direct reflection of rice commercialization (Zhu, 2017; Feng et al., 2017). Chalkiness is a typical quantitative trait in rice. The formation of chalkiness is controlled by many genes. In addition to genetic factors, it is also affected by the external environmental conditions during the filling process, such as sunshine intensity and temperature (Peng et al., 2016; Li, 2017; Zhu et al., 2017). The chalkiness area of rice was sensitive to the change of environment, and increased with the increase of light hours, daily average light and relative humidity (Li et al., 1989). Compared without chalkiness rice, the short chain amylopectin of chalky rice increased significantly, while the amylose and long chain amylopectin decreased significantly, and the transparency and milled rice rate decreased significantly, resulting in the reduction of cooking and eating quality and yield of rice (Peng et al., 2016; Kang et al., 2007). Genetic factors are the main cause of chalkiness formation, which is regulated by the genetic effect of parents and the interaction between heredity and environment (Zhang et al., 2017). At present, a large number of genes affecting rice chalkiness have been cloned from natural populations or mutants, such as OSPK2 (Cai, 2018), Chalk5 (Zhu, et al., 2017), OSPPDKB (Kang et al., 2014), GW2 (Song et al., 2007), SSIIIa (Fujita et al., 2007; Ryoo et al., 2007), flo2 (She et al., 2010) etc. The results of microstructural observation showed that chalkiness in endosperm was closely related to starch granule structure, arrangement and starch synthesis (Shen et al., 2000; Zhai et al., 1991). The structure and arrangement of starch granules is also an important character to determine the grain quality of rice. Chalkiness is formed by the opaque material refracted by light due to the round shape of composite starch granules, loose arrangement of starch and inflation between grains.

Giant embryo rice is a kind of special functional rice, whose embryo is 2-3 times larger than that of ordinary rice. The main nutrient elements in rice are concentrated in embryo, and its protein, mineral, vitamin, fat, $\gamma$-amino acid, folic acid and other nutrients are higher than that of ordinary rice (Zhang et al., 2007; Tang et al., 2020), which makes rice breeders pay more attention to the breeding of many excellent characters (Zheng et al., 2012; Guo et al., 2011). Previous studies mainly focused on gene molecular markers, gene location, growth dynamics, nutritional components and processing technology of giant embryo rice. The formation mechanism of chalkiness in endosperm of giant embryo rice is still unclear. In this study, the chalkiness of the endosperm of 16 rice varieties were detected and analyzed, and the endosperm cells and starch granules of the rice were observed by scanning electron microscopy. Thus, our results will lay a foundation for the breeding of new giant embryo rice varieties in the later stage.

\section{Materials and Methods}

\subsection{Materials}

All 16 giant embryo rice varieties originated from Xinyang Agriculture and Forestry University, all of which were non-glutinous. Among them, Baijupeishanghai, Juhongxuan-GB-19, Juhongxuanbai and Baijupei-GB-7 were indica rice varieties and the rest were japonica varieties (including Jujingzhan-GB-21, Jujingzhan-GB-20, Jujingzhan-GB-19, Jujingzhan-GB-12, Jujingzhan-GB-11, Jujingzhan-GB-7, Jujingzhan-GB-6, Jujingzhan-GB-5, Jujingzhan-GB-4, Jujingzhan-GB-3, Jujingzhan-GB-2, Jujingzhan-GB-2). Each giant embryo rice variety was planted in 2 rows with 12 plants in each row, and the row spacing was $16.5 \times 26.4 \mathrm{~cm}$. From sowing to final seed maturity, the test materials were managed by conventional cultivation in the rice experimental field of Xinyang Normal University. The rice grains were dried naturally after maturity and stored at room temperature for three months.

\subsection{Methodology}

\subsubsection{Determination of Chalkiness Character}

Cretaceous rate, chalkiness area and chalkiness degree of different giant embryo rice varieties were measured and analyzed according to the national standard GB/T17891-1999: 100 full and complete rice grains were randomly selected from each variety, and chalky rice grains were counted. According to the method of measuring chalkiness rate, the abdominal and cardiac white rates were determined (no back white grains were found), that is, 100 grains of fine rice were randomly selected from each variety, and the rice grains containing abdominal white and heart white were counted from them, repeated three times, and the average value was taken 
as the abdominal and heart white rates of each variety; chalkiness degree $=$ chalkiness rate $\times$ chalkiness area. SPSS software was used for the analysis of the measured data.

\subsubsection{SEM Observation}

The chalkiness grains were randomly selected from each giant embryo rice variety, and the back of the blade was tapped in the middle part of the rice to make it break. Then the fracture part was cut off with the blade, and about 2 3 mm thick samples were made. One part was observed by ordinary optical microscope, the other part was glued to the copper sample table with conductive adhesive. The HUS-5GB vacuum film plating instrument plated the section, then placed the sample under scanning electron microscope (RiLi S-4800) to observe the different parts of the grain section and take pictures.

\section{Results}

\subsection{Differences in Chalkiness Characters of Different Giant Embryo Rice Varieties}

The test results of the chalkiness traits of giant embryo rice varieties are shown in Figure 1. It can be seen that the chalkiness trait with the largest difference among varieties is chalkiness rate, followed by chalky area, and finally chalky degree. Juhongxuanbai, Jujingzhan-GB-12, Jujingzhan-GB-11 had the highest chalkiness rate, reaching 100.0\%, respectively. However, no chalkiness was observed in Jujingzhan-GB-7, Jujingzhan-GB-1, and the chalkiness rate was the lowest, only $2.0 \%$, respectively. The chalkiness rate of 15 giant embryo varieties varies between $2.0 \%$ and $100.0 \%$. The chalkiness degree varies between $0.4 \%$ and $21.0 \%$. The change trend is very similar to the chalkiness rate, Baijupei-GB-7, Juhongxuanbai, Jujingzhan-GB-12, Jujingzhan-GB-11, Jujingzhan-GB-6, Jujingzhan-GB-4 and Jujingzhan-GB-20 mainly occurs in the abdomen, and the rest of the varieties mainly occur in the center of the endosperm of rice. The variation range of the chalkiness area is between $9.4 \%$ and $56.6 \%$, and its change trend different from the chalkiness rate and chalkiness, chalkiness rate of Baijupeishanghai, Jujingzhan-GB-7 and Jujingzhan-GB-2 were not very high, but their chalkiness areas exceeded $30.0 \%$. It is worth noting that in the varieties of Jujingzhan-GB-7, Jujingzhan-GB-1, the existence of chalkiness traits is basically not observed, while Juhongxuanbai, Jujingzhan-GB-12, Jujingzhan-GB-11 varieties have a chalkiness rate of up to $100.0 \%$, and their chalkiness areas are $21.1 \%, 9.4 \%, 11.6 \%$, respectively; and chalkiness degree is $21.1 \%, 9.4 \%, 11.6 \%$, respectively. Further analysis shows that there is a clear linear relationship between chalkiness rate and chalkiness degree, and varieties with high chalkiness rate tend to have higher chalkiness degree.

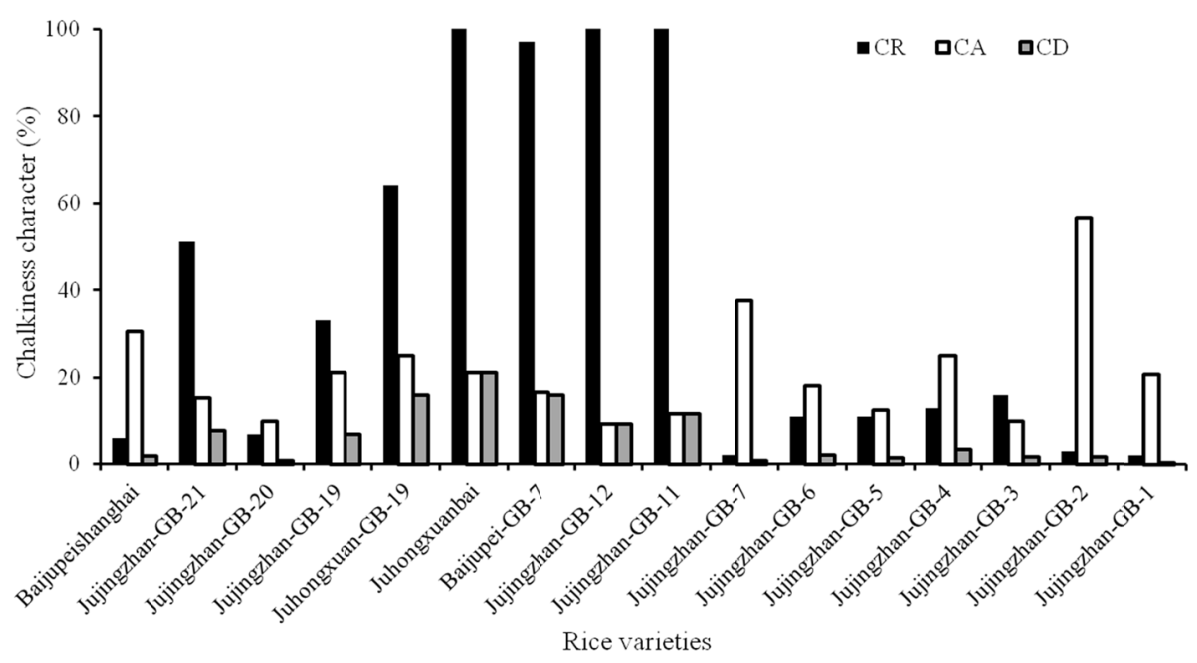

Figure 1. Chalkiness characters of the 16 giant embryo rice varieties

Note. $\mathrm{CR}$, chalkiness rate; $\mathrm{CA}$, chalkiness area; $\mathrm{CD}$, chalkiness degree.

\subsection{Endosperm Morphological Structure of Different Japonica Rice Varieties}

The representative polished rice was selected from 16 giant embryo rice varieties. The arrangement of endosperm cells, cell morphology, number of cell layers and the distribution of starch granules in cross section were observed by ordinary optical microscope and electron microscope scanning. The results are shown in Figure 2. The arrangement of endosperm cells in the cross section can be divided into four categories according 
to whether they are radiating from the center to the periphery and the obvious degree of radiating: category I is that there is obvious radiation from the center of rice cross section to the outer circumference, and the radiation is longer. In this case, there are varieties such as Jujingzhan-gb-21, Jujingzhan-gb-11, Baijupeiishanghai and so on, which are mainly that focus chalkiness on center (Figures $2 \mathrm{~A}$ and $2 \mathrm{E}$ ). Category II is also radiated from the center of the cross section of rice, but the endosperm cells arranged radially are not straight, or even some are bent together. In this case, there are mainly varieties such as Jujingzhan-GB-1, Jujingzhan-GB-19, Juhongxuanbai and so on, which are mainly that focus chalkiness on belly (Figures 2B and 2F). Although category III is radiated outwards along the center of the endosperm section of rice, and the radiation is not obvious, belongs to this kind of situation has Baijupei-GB-7, Jujingzhan-GB-12, Jujingzhan-GB-4 and the varieties with both focusing chalkiness on center and focusing chalkiness on belly (Figures $2 \mathrm{C}$ and $2 \mathrm{G}$ ). Category IV can hardly see any radiation from the center of the rice cut surface to the surroundings. In this case, there are varieties with low or no chalkiness, such as Jujingzhan-GB-7, Jujingzhan-GB-2 (Figures 2D and 2H).

Under the scanning electron microscope, the cell morphology of different japonica rice varieties can be clearly seen at a magnification of more than 2000 times. It can be seen that there are several layers of rectangular columnar endosperm cells in Jujingzhan-GB-21, Jujingzhan-GB-11, Baijupeishanghai and so on which are mainly composed of varieties of chalkiness in center, and there are many elliptical starch granules around which there are multiple layers of multilateral columnar cells (Figure 2A). There are several layers of oblong columnar endosperm cells in Jujingzhan-GB-1, Jujingzhan-GB-19, Juhongxuanbai and so on, which rice varieties mainly with abdominal white. And the non radiation state around the rest is evenly distributed starch granules, among which there are some multilateral irregular cells of different sizes (Figure 2B); Baijupei-GB-7, Jujingzhan-GB-12, Jujingzhan-GB-4 and so on varieties with both focusing chalkiness on center and focusing chalkiness on belly exist elliptical cells chimeric with each other to form irregular cell groups (Figure 2C). The polygonal columnar cells, elliptical cells and irregular cells of varieties with lower or no chalkiness such as Jujingzhan-GB-7 and Jujingzhan-GB-2 were not obvious or even almost absent (Figure 2D).
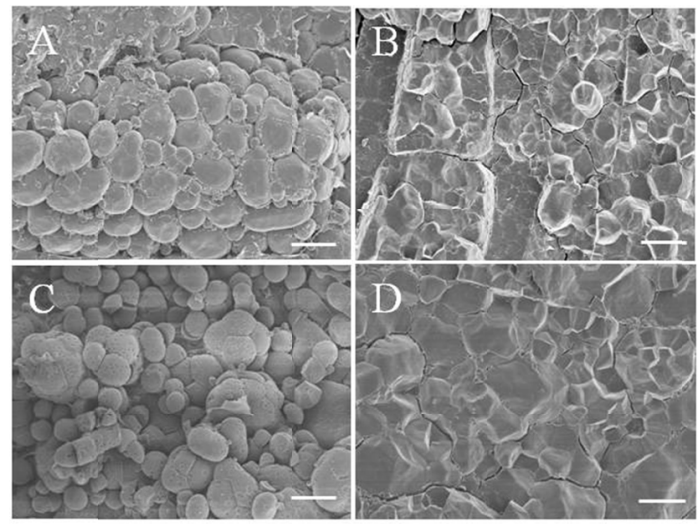

$\mathrm{E}$

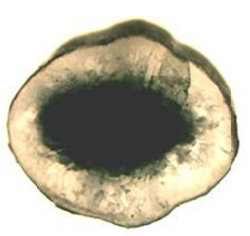

G

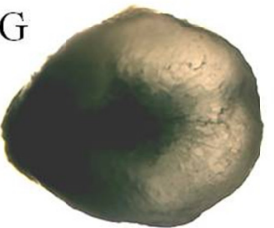

$\mathrm{F}$

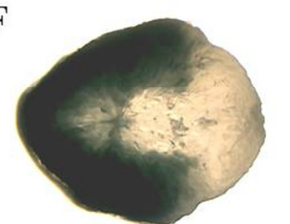

$\mathrm{H}$

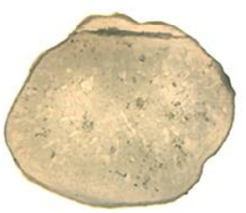

Figure 2. Low magnification view of the transversely fractured midregion of the tested rice

Note. A, E: Baijupeishanghai; B, F: Jujingzhan-GB-1; C, G: Jujingzhan-GB-12; D, H: Jujingzhan- GB-11; Bar = $40 \mu \mathrm{m}$. 
Observing the distribution of starch granules in the cut surface of rice samples by electron microscope, it can be seen that the distribution of starch granules of the giant embryo rice varieties with chalkiness traits is obviously uneven, and the starch granules were mainly distributed in the center and dorsal ventral diameter direction. The density of starch granules of different chalkiness giant embryo rice varieties is also different. The starch granules of chalkiness in the center giant embryo rice varieties are mainly distributed in the center, and the starch granules of white belly giant embryo rice varieties are mostly distributed in the belly of rice, while the starch granules of both chalkiness in the center and white belly giant embryo rice varieties are more and uneven in the center and belly of rice. The starch granules of giant embryonic rice cultivars without or with very low chalkiness rate were apparently relatively uniformly distributed throughout the cross section, and the density of starch granules was larger (Figure 2). The above results showed that there was a linear relationship between the structure and arrangement of endosperm cells on the cross section of rice samples and the distribution of starch granules on the cross section and the formation of chalkiness.

\subsection{Starch Granule Morphology of Chalky and Non-chalky Grain in Giant Embryo Rice Varieties}

The whole polished rice with chalkiness and the whole polished rice without chalkiness were selected from the above 16 varieties of giant embryo rice for scanning electron microscopy. The results showed that in the chalkiness part of rice there were spherical starch granules with no obvious edges and corners. The gap between two adjacent starch granules was relatively large. The starch granules were uneven in size, and most of them were single starch granules in free station, with small diameter and irregular accumulation. Starch granules have large clearance and loose arrangement (Figures 3A and 3D).

By comparing the starch granules of chalky rice and non-chalky rice, it is found that there is no obvious difference in the starch granules between them. Most starch granules are uniform in size, regular polygon or diamonds in shape, which are polyhedral corners, and starch granule gaps are small or no gaps (Figures 3B, 3C, $3 \mathrm{E}$, and $3 \mathrm{~F}$ ). The above results show that the formation of chalkiness in rice is closely related to the shape and arrangement of endosperm starch granules. The lower the degree of chalkiness, the better the starch granules develop, otherwise the starch granules do not develop well.
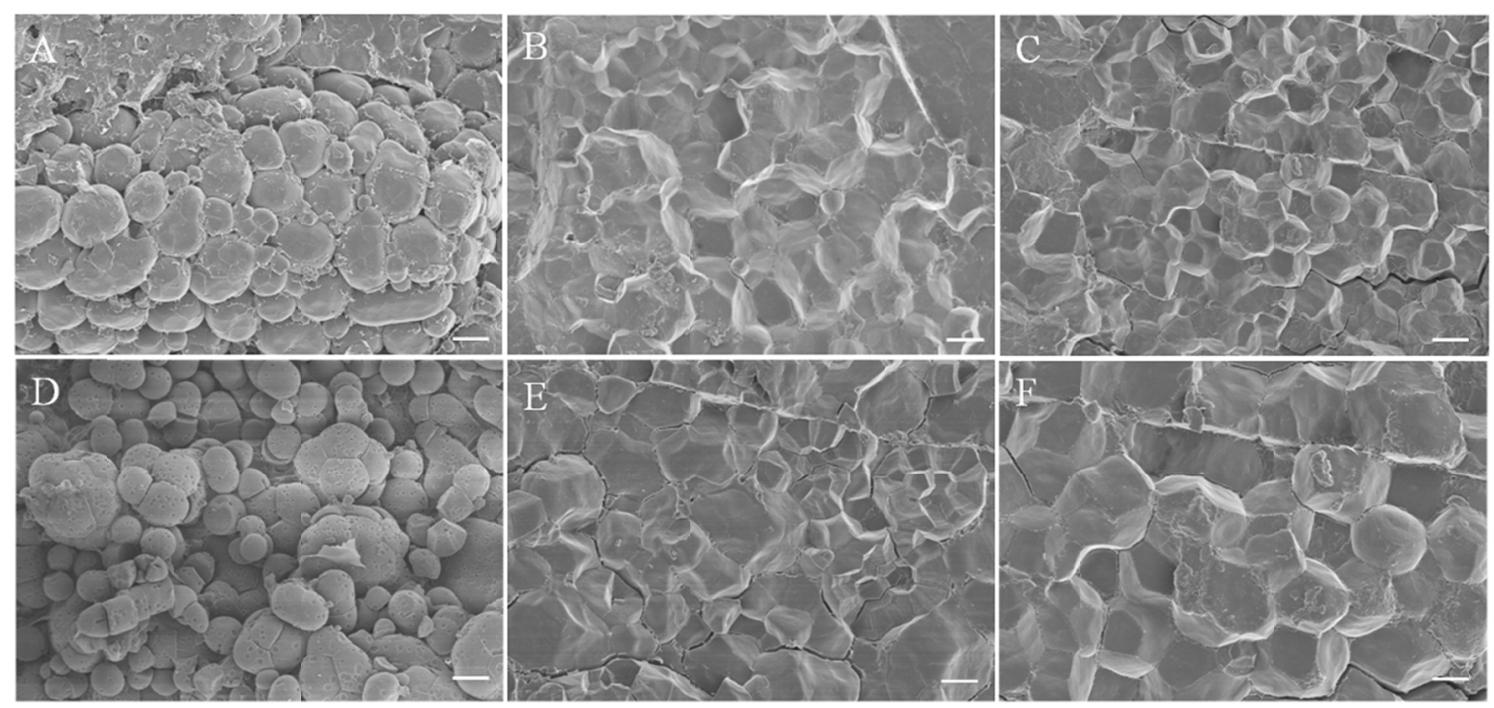

Figure 3. Scanning electron microscopic observation on starch granules in endosperm cells of the chalky and non-chalky rice

Note. A, D: the starch granules in the chalky rice; B, E: the starch granules in the transparent parts of rice; C, F: the starch granules in the non-chalky rice; $\mathrm{Bar}=20 \mu \mathrm{m}$. 

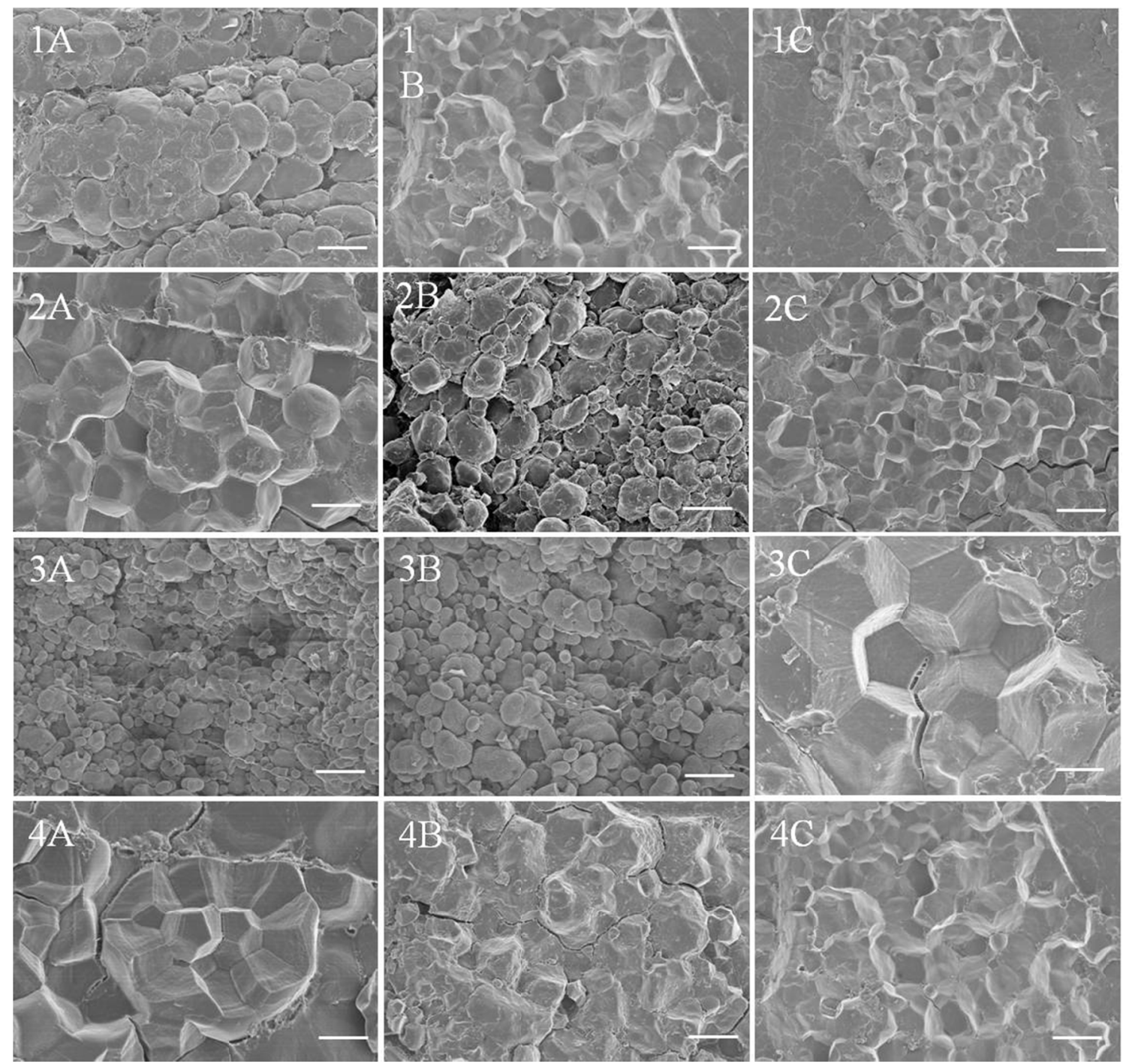

Figure 4. Scanning electron microscopic observation on starch granules in different part of the tested giant embryo rice varieties

Note. 1 4, Baijupeishanghai, Jujingzhan-GB-1, Jujingzhan-GB-12 and Jujingzhan-GB-2; A, starch granules in the center of the endosperm; $\mathrm{B}$, starch granules in the endosperm of the belly; $\mathrm{C}$, starch granules on the back of the endosperm; $\mathrm{Bar}=50 \mu \mathrm{m}$.

\subsection{Morphology of Starch Granules in Different Parts of Chalkiness Rice Varieties}

According to different parts of rice endosperm chalkiness, chalkiness can be divided into three types: white belly, white core and white back. The abdomen, center and back of chalkiness rice of 16 different giant embryo rice varieties were observed by scanning electron microscope. The results showed that the starch granules in the center of endosperm of Jujingzhan-GB-21, Jujingzhan-GB-11 and Baijupeishanghai varieties had relatively large space, loose arrangement and poor development of starch granules, while the starch granules in the back and abdomen of endosperm developed well (Figure 4-1). The starch granules in the abdomen of endosperm of Jujingzhan-GB-1, Jujingzhan-GB-19 and Juhongxuanbai are poorly developed, while the starch granules in the center and back of endosperm are well developed (Figure 4-2). The starch granules in the abdomen and center of endosperm of Baijupei-GB-7, Jujingzhan-GB-12 and Jujingzhan-GB-4 are arranged loosely, and the starch granules are poorly developed, while the starch granules on the back are well developed (Figure 4-3). The starch granules in the center, back and abdomen of endosperm of Jujingzhan-GB-7 and Jujingzhan-GB-2 were well developed (Figure 4-4). 


\section{Discussion}

\subsection{Distribution of Chalkiness and Starch Granules in Giant Embryo Rice}

Chalkiness not only affects the appearance quality of rice, but also affects the cooking and eating quality, milling quality and nutritional quality (Zheng et al., 2012; Liu et al., 2011; Yoshioka et al., 2007; Shen et al., 2007). The morphological structure of starch granules can be observed by scanning electron microscope, and the quality of rice can be judged preliminarily according to its morphological structure. The chalkiness was formed by oblong cells arranged in the direction of dorsal and ventral diameter (Zhai et al., 1991). The chalkiness was not only related to oblong cells, but also related to the arrangement and morphological results of starch granules in rice (Chang et al., 2006). In this experiment, through the observation of SEM, the starch granules of the varieties with higher chalkiness rate and chalkiness degree (Juhongxuanbai, Jujingzhan-GB-12 and Jujingzhan-GB-11) were obviously uneven in the cross section; the starch granules of the varieties with lower chalkiness rate and chalkiness degree (Jujingzhan-GB-7, Jujingzhan-GB-2, Baijupeishanghai, Jujingzhan-GB-2) were evenly distributed in the center of endosperm, abdomen and back. Through comprehensive analysis, we found that if the distribution of starch granules in the cross section is more uniform, the variety is not easy to produce chalkiness, and the corresponding chalkiness rate and chalkiness degree will be low or not. However, if the starch granules are distributed unevenly in the cross section and the gap between the starch granules is large, the arrangement is loose and the starch granules are poorly developed, which leads to the chalkiness easily, and the corresponding chalkiness rate and chalkiness degree will be higher. It is concluded that the starch granules distributed on the endosperm cross section of rice are closely related to the formation of chalkiness, which is consistent with previous studies.

\subsection{Development of Chalkiness and Starch Granules in the Same Giant Embryo Rice}

There are many granular starch granules in every starch body of rice, most of which are in the state of starch complex. Previous studies have found that the size of starch granules in the chalkiness part of rice is relatively smaller than that in the non chalkiness part (Zhao, 2015). By scanning electron microscopy, it was found that the starch granules in the endosperm of rice had different morphological structure and arrangement in different parts of the same giant embryo rice. By scanning electron microscopy, it was found that the starch granules in the endosperm of rice grain were also different in their morphological structure and arrangement in different parts of the same giant embryo rice. Compared with the non chalky part, there were spherical starch granules with different sizes in the chalky part of rice, and most of them were single starch granules in the free state. The arrangement of starch granules was loose and the gap was large, so they could not develop into starch granule complex produces chalkiness. If the starch granules in rice are closely arranged in a complex state, it is not easy to produce chalkiness.

Previous studies have found that most of the chalkiness traits tend to grow in the middle or abdomen of rice, with little or no white back trait (Ying et al., 2019; Peng et al., 2016a; Peng et al., 2016b; Kang et al., 2003). There is a certain correlation between the formation of starch granule morphology and chalkiness phenotype. Among the endosperm of 16 giant embryo rice varieties in this study, no white back character was found, and the starch granules on the back of rice grains were regular rhombus or regular and other multilateral, angular. The starch granules had little or no gap, which indicated that the starch granules on the back of rice grain developed well. The reason may be related to the transport mode of nutrients, which were generally transported to the back first to the center and abdomen. It can be seen that the chalkiness of giant embryo rice is closely related to starch development.

\subsection{The Cause of Chalkiness in Giant Embryo Rice}

Chalkiness is a complex quantitative trait in rice, which is not only controlled by many genes, but also related to genetic factors, starch granule structure, arrangement and external environment (Peng et al., 2016a; Li, 2017; Zhu et al., 2017). Based on the previous research and the 16 giant embryo rice varieties in this experiment, there are obvious differences in chalkiness characters of rice endosperm, indicating that the chalkiness characters of rice are mainly controlled by genetic factors. However, the formation of chalkiness is a very complex process, and the generation of chalkiness may be related to the "source", "sink" and "flow" in rice plants. If any of them changes or they are abnormal, it may lead to the occurrence of rice chalkiness (Yan et al., 2003). Therefore, it can be inferred from the results of this study that the occurrence and development of starch granules in rice endosperm and the precipitation of starch granules in the later stage are closely related to the formation of rice chalkiness. However, the distribution of starch granules, the early generation, the development process, the later deposition mode, this series of regulatory ways, how many genes are involved in the formation of chalkiness, need further research. 


\section{Conclusion}

By observing the microstructure of chalkiness characters (including chalkiness rate, chalkiness degree and chalkiness area) of the 16 giant embryo rice varieties, it was found that the chalkiness characters of different giant embryo rice endosperm were closely related to the morphological structure and arrangement of starch granules in endosperm cells. In different parts of the endosperm of the same giant embryo rice variety, there were obvious differences in chalkiness characters and starch granules in endosperm cells. Therefore, our results will provide an important reference for the improvement of chalkiness character and the breeding of new rice varieties.

\section{Acknowledgements}

This work was financially supported by National Natural Science Foundation of China (U1604110, 31600992, 31801332), Key Project of Science and Technology in Henan Province (192102110119, 182102110442), The Training Plan of Young Backbone Teachers in Colleges and Universities of Henan Province (2019GGJS162), Postgraduate Education Reform Project of Henan Province (2019SJGLX088Y), Key Scientific Research Projects of Universities in Henan Province (19A180030), Nanhu Scholars Program for Young Scholars of XYNU (2016054), Innovative Training Program of University Students in Henan Province (201910477004).

\section{References}

Bowles, D. (2012). Towards increased crop productivity and quality. Current Opinion in Biotechnology, 23(2), 202-203. https://doi.org/10.1016/j.copbio.2011.12.014

Cai, Y. C. (2018). Mapping cloning and functional study of chalky rice gene OsPK2. Beijing: Chinese Academy of Agricultural Sciences.

Chang, H. Y., Kang, H. Q., Fu, H. L., Tang, W. W., Deng, Y., Liu, J., \& Lan, L. Q. (2006). Study on chalkiness difference and endosperm cytology structure of different rice varieties (lines).

Dan, W. H. (2019). QTLs mapping of appearance quality and starch grain shape in rice. Huazhong Agricultural University, China.

Feng, F., Li, Y. J., Qin, X. L., Yun, C. L., \& Siddique, K. H. M. (2017). Changes in rice grain quality of indica and japonica type varieties released in China from 2000 to 2014. Front Plant Sci, 8, 1863. https://doi.org/ 10.3389/fpls.2017.01863

Fernie, A., Tadmor, Y., \& Zamir, D. (2006) Natural genetic variation for improving crop quality. Current Opinion in Plant Biology, 9(2), 196-202. https://doi.org/10.1016/j.pbi.2006.01.010

Fitzgerald, M., Mccouch, S., \& Hall, R. (2009). Not just a grain of rice: The quest for quality. Trends in Plant Science, 14(3), 133-139. https://doi.org/10.1016/j.tplants.2008.12.004

Hu, K. F., Yang, Z. M., Zhu, Y. G., \& Lei, Z. S. (2003). Research Progress on the correlation between chalkiness and rice quality. Hubei Agricultural Sciences, 19-22.

Kang, H. G., Park, S., \& Matsuoka, M. (2005). White-core endosperm floury endosperm-4 in rice is generated by knockout mutations in the C4-type pyruvate orthophosphate dikinase gene (OSPPDKB). The Plant Journal, 42(6), 901-911. https://doi.org/10.1111/j.1365-313X.2005. 02423.x

Kang, H. Q., \& Chang, H. Y. (2007). The chalky characters and endosperm structure of main parent materials of hybrid rice were observed by electron microscopy. Chinese Agricultural Science Bulletin, 23(4), 180-185.

Kim, K. S., Kang, H. J., Hwang, I. K., Hwang H. G., Kim T. Y., \& Choi, H. C. (2004). Comparative structure of ilpumbyeo, a high-quality Japonica rice, and its mutant suweon 464: Scanning and transmission electron microscopy studies. Journal of Agricultural and Food Chemistry, 52(12), 3876-3883. https://doi.org/ $10.1021 / \mathrm{jf} 049767 \mathrm{r}$

Li, B. Q. (2017). Effect of Rice Chalkiness on rice quality and its improvement. Anhui Agricultural Science Bulletin, 23(6), 56-58.

Li, X., Gu, H. M., \& Pan, X. B. (1989). Study on the quality of rice II. The influence of environmental conditions on the quality of rice during filling. Jiangsu Journal of Agricultural Sciences, 10(1), 7-12.

Liu, X. L., Wan, X. Y., Ma, X. D., \& Wang, J. M. (2011). Dissecting the genetic basis for the effect of rice chalkiness, amylose content, protein content, and rapid viscosity analyzer profile characteristics on the eating quality of cooked rice using the chromosome segment substitution line population across eight environments. Genome, 54, 64-80. https://doi.org/10.1139/G10-070 
Peng, B., Pang, R. H., Sun, Y. F., Geng, L. P., Song, X. H., Li, H. L., ... Song, S. Z. (2016a). Study on chalky characters of endosperm of fragrant rice and observation by scanning electron microscope. Journal of Southern Agriculture, 47(10), 1635-1641.

Peng, B., Sun, Y. F., Pang, R. H., Li, H. L., Song, X. H., Yuan, H. Y., ... Song, S. Z. (2016b). Study on chalkiness characters and endosperm structure of different japonica rice varieties. Acta Agriculturae Zhejiangensis, 28(11), 1803-1811.

Shen, X. P., Shen, X. Y., Gu, L., Gong, L. P., \& Zhang, H. C. (2007). Effect of seeding time on chalkiness of liangyoupeijiu in Jiangsu rice growing areas at different latitudes. Chinese Journal of Rice Science, 21, 677-680. https://doi.org/10.16819/j.1001-7216.2007.06.020

Sheng, B. (2000). Electron microscopic observation on the development of mesoendosperm starch grains in chalkiness formation of dry indica rice. Chinese Journal of Rice Science, 14(4), 225-228.

Siebenmorgen, T., Grigg, B., \& Lanning, S. (2013). Impacts of preharvest factors during kernel development on rice quality and functionality. Annual Review of Food Science and Technology, 4(4), 101-115. https://doi.org/10.1146/annurev-food-030212-182644

Sing, H. N., Sodehi, N. S., Kaur, M., Manmeet, K., \& Saxena, S. K. (2003). Physicochemical, morphological, thermal, cooking and textural properties of chalky and translucent rice kernels. Food Chemistry, 82(3), 433-439. https://doi.org/10.1016/s0308-8146(03)00007-4

Tang, S. X., Jiang, Y. Z., Yv, H. Y., Zhang, Y. K., \& Li, S. S. (1999). Scanning electron microscopic observation of amyloplast in early indica endosperm. Acta Agronomica Sinica, 25(2), 269-271. https://doi.org/11.1809/ s25.2.1999.269

Xing, Y., \& Zhang, Q. (2010). Genetic and molecular bases of rice yield. Annual Review of Plant Biology, 61(1), 421-442. https://doi.org/10.1146/annurev-arplant-042809-112209

Ying, Y. N., Pang, R. H., \& Bao, J. S. (2019). The role of embryomastoid variants in the regulation of starch synthesis in rice. Journal of Nuclear Agricultural Sciences, 33(12), 2362-2375.

Yoshioka, Y., Iwata, H., Tabata, M., Ninomiya, S., \& Ohsawa, R. (2007). Chalkiness in rice: Potential for evaluation with image analysis. Crop Science, 47, 2113-2120. https://doi.org/10.2135/cropsci2006. $10.0631 \mathrm{sc}$

Zhang, Q. F. (2007). Strategies for developing Green Super Rice. Proceedings of the National Academy of Sciences of the United States of America, 104(104), 16402-16409. https://doi.org/10.1073/pnas.0708013104

Zhang, Q. Q., Zhang S. B., Huang, R. H., \& Zheng, B. D. (2009). Analysis of nutritional components of megaembryo functional rice. Journal of Nuclear Agricultural Sciences, 23(5), 833-838.

Zhang, X. C. (2017). Identification of two chalkiness mutants and map cloning of mutant gene. Beijing: Chinese Academy of Agricultural Sciences.

Zhang, Y. H., Zheng, Z., Chen, Y. Y., Huang, R. H., Zheng, B. D., \& Zhang, Q. Q. (2013). Analysis of nutritional components of new lines of giant embryo rice. Journal of Nuclear Agricultural Science, 27(9), 1331-1336.

Zhao, H., Wang, J. M., Zhang, Q. F., Zhao, Q., Mei, S. F., Liu, X. L., \& Cheng, F. M. (2015). Key enzyme activity of starch synthesis and its relationship with physicochemical properties of starch in rice during grain filling with sug-11 mutant of glycosperm. Chinese Journal of Rice Science, 29(1), 73-81.

Zheng, L. N., Zhang, W. W., Liu, S. J., Chen, L. M., Liu, X., Chen, X. G., ... Wan, J. M. (2012). Genetic relationship between grain chalkiness, protein content, and paste viscosity properties in a backcross inbred population of rice. Journal of Cereal Science, 56, 153-160. https://doi.org/10.1016/j.jcs.2012.05.003

Zheng, Z., Huang, Z. C., Zhang, Y. H., Huang, R. H., \& Zhang, Q. Q. (2012). Progress in research on giant embryo rice. Subtropical Agriculture Research, 8(4), 221-225.

Zhu, J. Y., Wang, Q. Q., Wang, J., Fan, F. J., Li, W. Q., Wang, F. Q., .. Yang, J. (2017). Development and application of chalky gene Chalk5 functional marker in rice. Acta Agriculturae Boreali-Sinica, 32(1), 1-8. 


\section{Copyrights}

Copyright for this article is retained by the author(s), with first publication rights granted to the journal.

This is an open-access article distributed under the terms and conditions of the Creative Commons Attribution license (http://creativecommons.org/licenses/by/4.0/). 